\title{
Shock Waves and Other Solutions to the Benjamin-Bona-Mahoney-Burgers Equation with Dual Power-Law Nonlinearity
}

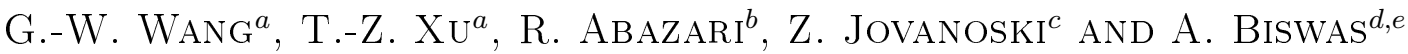 \\ ${ }^{a}$ School of Mathematics and Statistics, Beijing Institute of Technology, Beijing-100081, People's Republic of China \\ ${ }^{b}$ Young Researchers and Elite Club, Ardabil Branch, Islamic Azad University, Ardabil, Iran \\ ${ }^{c}$ Applied and Industrial Mathematics Research Group, School of Physical, \\ Environmental and Mathematical Sciences, UNSW Canberra, ACT 2600, Australia \\ ${ }^{d}$ Department of Mathematical Sciences, Delaware State University, Dover, DE 19901-2277, USA \\ e Department of Mathematics, Faculty of Sciences, King Abdulaziz University, Jeddah-21589, Saudi Arabia
}

(Received April 22, 2014)

We study the hybrid Benjamin-Bona-Mahoney-Burgers equation with dual power-law nonlinearity. Three different techniques - the ansatz method, Lie-symmetry analysis and the $\left(G^{\prime} / G\right)$-expansion method - are used to find shock wave solutions. Several constraint conditions naturally emerge that guarantee the existence of shock waves. We discuss the nature of the solutions generated by the different methods.

DOI: 10.12693 /APhysPolA.126.1221

PACS: 02.30.Ik; 02.30.Jr; 02.20.Qs

\section{Introduction}

There are several forms of nonlinear evolution equations that are studied in the areas of applied mathematics, theoretical physics and in the engineering sciences [1-26]. Of particular interest is the dynamics of shallow water waves governed by certain nonlinear evolution equations. In this context, one frequently encounters the well known Kortweg-de Vries (KdV) equation, Peregrine equation, Kawahara equation, Gardner equation, Gear-Grimshaw equation and the Bona-Chen equation. These models exhibit single layered as well as two-layered fluid flow on lakes and shores.

Another well-known model, the Benjamin-BonaMahoney (BBM) equation, models the dynamics of shallow water waves in the presence of advection. The model considered in this paper extends the BBM equation by including the so-called dual-power law nonlinearity and is augmented by the explicit presence of a dissipative perturbation term. The inclusion of these additional features leads to the hybrid dissipative equation referred to as the Benjamin-Bona-Mahoney-Burgers (BBM-B) equation. The primary aim of this paper is to utilise three different approaches to find exact shock wave solutions, also known as topological soliton solutions, to the BBM-B equation.

\section{Governing equation}

The BBM-B equation that is studied is $[5,8]$ :

$u_{t}+a u_{x}+\left(b_{1} u^{n}+b_{2} u^{2 n}\right) u_{x}+c u_{x x}+k u_{x x t}=0$. (1) The wave profile is represented by $u(x, t)$. The coefficient $a$ represents the strength of advection or drifting, while $b_{1}$ and $b_{2}$ measure the strengths of the two nonlinear terms with the exponent $n$ being the power law nonlinearity parameter. Finally, the parameters $c$ and $k$ are the dissipative diffraction coefficient and the higherorder spatio-temporal dispersion coefficient, respectively. Thus Eq. (1) models the shallow water wave flow on lakes and beaches with a dissipative factor that is given by the coefficient $c$.

Equation (1) is not integrable by the inverse scattering transform. Therefore we resort to other integration tools that are fully discussed in the sections that follow. These mathematical tools include the ansatz method, the Liesymmetry approach and the $G^{\prime} / G$-expansion method. Utilising these three integration architectures we find exact 1-soliton solutions and derive parameter constraint equations that are necessary to ensure the existence of these solutions.

\section{Ansatz approach}

This section focuses on extracting the shock wave solution to Eq. (1). The starting point is to select the travelling wave ansatz given by

$$
u(x, t)=A \tanh ^{p} \tau, \quad \tau=H(x-\lambda t) .
$$

Here, the shock wave is described by the four parameters $A, H, \lambda$ and $p$. The parameters $A$ and $H$ represent, respectively, the dilation factor (amplitude) and the steepening factor of the wave, whereas $\lambda$ is the speed of the wave. The shape parameter $p$ is determined upon the application of the balancing principle.

$$
\begin{aligned}
& \text { Substituting (2) into (1) gives } \\
& \begin{array}{l}
(a-\lambda)\left(\tanh ^{p-1} \tau-\tanh ^{p+1} \tau\right) \\
\quad+b_{1} A^{n}\left(\tanh ^{n p+p-1} \tau-\tanh ^{n p+p+1} \tau\right) \\
\quad+b_{2} A^{2 n}\left(\tanh ^{2 n p+p-1} \tau-\tanh ^{2 n p+p+1} \tau\right) \\
\quad+c H\left\{(p-1) \tanh ^{p-2} \tau-2 p \tanh ^{p} \tau+(p+1) \tanh ^{p+2} \tau\right\}
\end{array}
\end{aligned}
$$




$$
\begin{aligned}
& -k \lambda H^{2}\left[(p-1)(p-2) \tanh ^{p-3} \tau\right. \\
& -\left\{2 p^{2}+(p-1)(p-2)\right\} \tanh ^{p-1} \tau \\
& +\left\{2 p^{2}+(p+1)(p+2)\right\} \tanh ^{p+1} \tau \\
& \left.-(p+1)(p+2) \tanh ^{p+3} \tau\right]=0 .
\end{aligned}
$$

By the balancing principle, equating the exponents $2 n p+$ $p+1$ and $p+3$ leads to $p=1 / n$. It should be noted that the same result is arrived at when $2 n p+p-1$ and $p+1$ are equated to each other. Further, there are two standing alone perturbation terms expressed through $\tanh ^{p-2} \tau$ and $\tanh ^{p-3} \tau$. Therefore, setting their coefficients to zero leads to the solution $p=1$, which immediately implies that $n=1$. Thus, the BBM-B equation with dual power-law nonlinearity reduces to the BBM-B equation with dual nonlinearity, thus (1) can now be recasted as

$$
u_{t}+a u_{x}+\left(b_{1} u+b_{2} u^{2}\right) u_{x}+c u_{x x}+k u_{x x t}=0 .
$$

Finally, setting the coefficients of the remaining linearly independent functions $\tanh ^{j} \tau,(j=0,1,2,3,4)$ in (3) to zero leads to

$$
\begin{aligned}
& \lambda=\frac{a}{1-2 k H^{2}}, \\
& \lambda=\frac{a-b_{2} A^{2}}{1-8 k H^{2}}, \\
& b_{1} A=2 c H, \\
& b_{2} A^{2}=6 \lambda k H^{2} .
\end{aligned}
$$

Now, from (5) and (6), equating the two values of the speed $\lambda$ gives

$$
b_{2} A^{2}-2 k b_{2} A^{2} H^{2}-6 a k H^{2}=0 .
$$

In terms of the model parameters $a, b_{1}, b_{2}, c$ and $k$, the wave parameters are

$$
\begin{aligned}
& A=\frac{1}{b_{1}} \sqrt{\frac{2 b_{2} c^{2}-3 a b_{1}^{2} k}{k b_{2}},} \\
& H=\sqrt{\frac{2 b_{2} c^{2}-3 a b_{1}^{2} k}{4 k b_{2} c^{2}},} \\
& \lambda=\frac{2 b_{2} c^{2}}{3 b_{1}^{2} k},
\end{aligned}
$$

which completely characterises the topological 1-soliton solution.

\section{Lie-symmetry analysis}

We now turn to the Lie-symmetry architecture to construct the solution to the BBM-B Eq. (1).

\subsection{Constructing infinitesimal generators}

In this section we perform a Lie group analysis of (1). We assume (1) is invariant under a one-parameter Lie group of point transformations

$$
\begin{aligned}
& t^{*}=t+\epsilon \tau(x, t, u)+\mathrm{O}\left(\epsilon^{2}\right), \\
& x^{*}=x+\epsilon \xi(x, t, u)+\mathrm{O}\left(\epsilon^{2}\right), \\
& u^{*}=u+\epsilon \eta(x, t, u)+\mathrm{O}\left(\epsilon^{2}\right),
\end{aligned}
$$

$$
V=\tau(x, t, u) \frac{\partial}{\partial t}+\xi(x, t, u) \frac{\partial}{\partial x}+\eta(x, t, u) \frac{\partial}{\partial u} .
$$

If the vector field (14) generates a symmetry of (1), then $V$ must satisfy the Lie invariance condition

$$
\left.\operatorname{pr}^{(3)} V(\Delta)\right|_{\Delta=0}=0 \text {, }
$$

where $\Delta=u_{t}+a u_{x}+\left(b_{1} u^{n}+b_{2} u^{2 n}\right) u_{x}+c u_{x x}+k u_{x x t}$. Applying the third prolongation $\mathrm{pr}^{(3)} V$ to (18), we find the following system of symmetry equations:

$$
\begin{aligned}
& \eta^{t}=D_{x}(\eta)-u_{x} D_{x}(\xi)-u_{t} D_{x}(\tau)= \\
& D_{x}\left(\eta-\xi u_{x}-\tau u_{t}\right)+\xi u_{x x}+\tau u_{x t}= \\
& \eta_{x}+\left(\eta_{u}-\xi_{x}\right) u_{x}-\tau_{x} u_{t}-\xi_{u} u_{x}^{2}-\tau_{u} u_{x} u_{t}, \\
& \eta^{t}=D_{t}(\eta)-u_{x} D_{t}(\xi)-u_{t} D_{t}(\tau)= \\
& D_{t}\left(\eta-\xi u_{x}-\tau u_{t}\right)+\xi u_{x t}+\tau u_{t t}= \\
& \eta_{t}-\xi_{t} u_{x}+\left(\eta_{u}-\tau_{t}\right) u_{x}-\tau_{t} u_{t}-\xi_{u} u_{x} u_{t}-\tau_{u} u_{t}^{2}, \\
& \eta^{x x}=D_{x}\left(\eta^{x}\right)-u_{x t} D_{x}(\tau)-u_{x x} D_{x}(\xi) \ldots, \\
& \eta^{x x x}=D_{x}\left(\eta^{x x}\right)-u_{x x t} D_{x}(\tau)-u_{x x x} D_{x}(\xi) \ldots, \\
& \eta^{x x t}=D_{t}\left(\eta^{x x}\right)-u_{x x t} D_{t}(\tau)-u_{x x x} D_{t}(\xi) \ldots,
\end{aligned}
$$

and the invariant condition now reads

$$
\begin{gathered}
\eta^{t}+a \eta^{x}+b_{1} u^{n} \eta^{x}+b_{2} u^{2 n} \eta^{x}+b_{1} n \eta u_{x} u^{n-1} \\
+2 b_{2} n \eta u_{x} u^{2 n-1}+c \eta^{x x}+k \eta^{x x t}=0 .
\end{gathered}
$$

Here, $D_{i}$ denotes the total derivative operators

$$
D_{i}=\frac{\partial}{\partial x^{i}}+u_{i} \frac{\partial}{\partial u}+u_{i j} \frac{\partial}{\partial u_{j}}+\cdots, \quad i=1,2,
$$

and $\left(x^{1}, x^{2}\right)=(t, x)$.

Then, in terms of the Lie-symmetry analysis, one can get

$$
\tau=c_{1}, \quad \xi=c_{2}, \quad \eta=0,
$$

where $c_{1}$ and $c_{2}$ are arbitrary constants. Equation (1) admits the two-dimensional Lie algebra, called the principle algebra, with basic operators [11]:

$$
V_{1}=\frac{\partial}{\partial t}, \quad V_{2}=\frac{\partial}{\partial x} .
$$

It is easy to check that the symmetry generators form a closed Lie algebra

$$
\left[V_{1}, V_{2}\right]=0, \quad\left[V_{2}, V_{1}\right]=0 \text {. }
$$

To obtain the group transformation generated by the infinitesimal generators $V_{1}$ and $V_{2}$, we solve the following initial value problems:

$$
\begin{array}{ll}
\frac{\mathrm{d}(\bar{x}(\varepsilon))}{\mathrm{d} \varepsilon}=\xi(\bar{x}(\varepsilon), \bar{t}(\varepsilon), \bar{u}(\varepsilon)), & \bar{x}(0)=x, \\
\frac{\mathrm{d}(\bar{t}(\varepsilon))}{d \varepsilon}=\tau(\bar{x}(\varepsilon), \bar{t}(\varepsilon), \bar{u}(\varepsilon)), & \bar{t}(0)=t, \\
\frac{\mathrm{d}(\bar{u}(\varepsilon))}{\mathrm{d} \varepsilon}=\eta(\bar{x}(\varepsilon), \bar{t}(\varepsilon), \bar{u}(\varepsilon)), & \bar{u}(0)=u,
\end{array}
$$

where $\varepsilon$ is a small parameter. So, we obtain the Liesymmetry group

with infinitesimal generator 


$$
g:(x, t, u) \rightarrow(\bar{x}, \bar{t}, \bar{u}) .
$$

Exponentiating the infinitesimal symmetries of (1), we get the one-parameter groups $g_{i}(\varepsilon)$ generated by $V_{i}(i=$ $1,2)$ :

$$
\begin{aligned}
& g_{1}:(x, t, u) \mapsto(x, t+\varepsilon, u), \\
& g_{2}:(x, t, u) \mapsto(x+\varepsilon, t, u) .
\end{aligned}
$$

The symmetry groups $g_{1}$ and $g_{2}$ demonstrate the timeand space-invariance of the equation. Consequently, we obtain the Theorem

Theorem 1 If $u=f(x, t)$ is a solution of third-order equation (1), so are the functions

$$
\begin{aligned}
& g_{1}(\varepsilon) \cdot f(x, t)=f(x-\varepsilon, t), \\
& g_{2}(\varepsilon) \cdot f(x, t)=f(x, t-\varepsilon) .
\end{aligned}
$$

\subsection{Symmetry reductions and exact group invariant solutions}

By balancing the nonlinear term with that of the highest order partial derivative in (1), similar to that of the ansatz method discussed above, we make the transformation

$$
u(x, t)=f(\xi)^{\frac{1}{n}},
$$

where $f(\xi)$ is a non-zero real-valued function of the single independent variable $\xi$.

\subsubsection{Infinitesimal generator $V_{1}$}

The group-invariant solution corresponding to $V_{1}$ where $\xi=t$ is the group-invariant is obtained by substituting (21) into (1) and leads to $f^{\prime}(\xi)=0$. Upon integrating, leads to the trivial solution $u(x, t)=C$, where $C$ is a constant.

\subsubsection{Infinitesimal generator $V_{2}$}

For the generator $V_{2}$, where $\xi=x$ is the groupinvariant, reduces (1) to the ordinary differential equation (ODE):

$$
n\left(a+b_{1} f+b_{2} f^{2}\right) f f^{\prime}+c(1-n)\left(f^{\prime}\right)^{2}+c n f f^{\prime \prime}=0,
$$

where $f^{\prime}$ is the derivative with respective to $\xi$.

\subsubsection{Infinitesimal generator $V_{1}+\lambda V_{2}$}

For the linear combination $V_{1}+\lambda V_{2}$, where $\xi=x-\lambda t$ is the group-invariant, leads to the ODE:

$$
\begin{aligned}
& n^{2}\left(a-\lambda+b_{1} f+b_{2} f^{2}\right) f^{2} f^{\prime}+c n(1-n) f\left(f^{\prime}\right)^{2} \\
& \quad+n^{2} c f^{2} f^{\prime \prime}-k \lambda(1-n)(1-2 n)\left(f^{\prime}\right)^{3} \\
& \quad-3 n k \lambda(1-n) f f^{\prime} f^{\prime \prime}-n^{2} k \lambda f^{2} f^{\prime \prime \prime}=0 .
\end{aligned}
$$

If $\lambda \neq 0$, then the solution to (22) is in the form of a travelling wave with speed $\lambda$.

By applying the generalised algebraic method to (22), one can obtain explicit travelling wave solutions [6]. Because the detailed description of the method can be found in [6], we omit it here and consider its application to the BBM-B equation with dual power-law nonlinearity.

Suppose that (22) can be written as

$$
f(\xi)=\sum_{i=0}^{p} a_{i} \varphi^{i}(\xi),
$$

where $a_{i}(i=0, \ldots, p)$ are constants to be determined for some positive integer $p$ and that the functions $\varphi(\xi)$ satisfy the Riccati equation

$$
\varphi^{\prime}=A+B \varphi+C \varphi^{2} .
$$

By balancing terms in (22), we get $p=1$, so that in this case

$$
f(\xi)=a_{0}+a_{1} \varphi .
$$

Substituting (25) into (22) and using (24), then collecting coefficients of monomials of $\varphi$ with the aid of MAPLE, followed by setting each coefficient to zero, we get for any positive $n$ the following parameter values:

$$
\begin{aligned}
& a=\frac{k \lambda\left(B a_{1}-2 C a_{0}\right)^{2}-(B c-n \lambda) n a_{1}^{2}+2 C c n a_{0} a_{1}}{n^{2} a_{1}^{2}}, \\
& A=\frac{a_{0}\left(B a_{1}-C a_{0}\right)}{a_{1}^{2}}, \\
& b_{1}=\frac{C k \lambda(n+1)(n+2)\left(B a_{1}-2 C a_{0}\right)-n(n+1) C c a_{1}}{n^{2} a_{1}^{2}}, \\
& b_{2}=\frac{C^{2} k \lambda(n+1)(2 n+1)}{n^{2} a_{1}^{2}} .
\end{aligned}
$$

where $B, C, c, k, a_{0}, a_{1}, \lambda$ are arbitrary constants. The solutions read

$$
\begin{aligned}
& f(\xi)=\frac{\Delta a_{1}}{2 C}\left[1-\tanh \left(\frac{\Delta}{2} \xi\right)\right], \\
& f(\xi)=\frac{\Delta a_{1}}{2 C}\left[1-\operatorname{coth}\left(\frac{\Delta}{2} \xi\right)\right], \\
& f(\xi)=\frac{\Delta a_{1}}{2 C}[1-(\tanh (\Delta \xi) \pm i \operatorname{sech}(\Delta \xi))], \\
& f(\xi)=\frac{\Delta a_{1}}{2 C}[1-(\operatorname{coth}(\Delta \xi) \pm \operatorname{csch}(\Delta \xi))], \\
& f(\xi)=\frac{\Delta a_{1}}{4 C}\left[2-\left(\tanh \left(\frac{\Delta}{4} \xi\right)+\operatorname{coth}\left(\frac{\Delta}{4} \xi\right)\right)\right] . \\
& f(\xi)=\frac{\Delta a_{1}}{2 C}\left[1+\frac{\gamma_{+}-E \cosh (\Delta \xi)}{F+E \sinh (\Delta \xi)}\right], \\
& f(\xi)=\frac{\Delta a_{1}}{2 C}\left[1-\frac{\gamma_{-}+E \sinh (\Delta \xi)}{F+E \cosh (\Delta \xi)}\right], \\
& f(\xi)=a_{0}\left[1-\frac{\Delta-B}{\Delta \tanh \left(\frac{\Delta}{2} \xi\right)-B}\right] \text {, } \\
& f(\xi)=a_{0}\left[1-\frac{\Delta-B}{\Delta \operatorname{coth}\left(\frac{\Delta}{2} \xi\right)-B}\right] \text {, } \\
& f(\xi)=a_{0}\left[1-\frac{\Delta-B}{\Delta \tanh (\Delta \xi)-B \pm i \Delta \operatorname{sech}(\Delta \xi)}\right] \\
& f(\xi)=a_{0}\left[1-\frac{\Delta-B}{\Delta \operatorname{coth}(\Delta \xi)-B \pm \Delta \operatorname{csch}(\Delta \xi)}\right]
\end{aligned}
$$

with $\Delta=\left(2 C a_{0}-B a_{1}\right) / a_{1}>0, \gamma_{ \pm}=\sqrt{F^{2} \pm E^{2}}$, where $F$ and $E$ are two non-zero real constants satisfying $F^{2}-E^{2}>0$. Additional solutions can be obtained in terms of trigonometric functions under the substitution $\Delta \rightarrow i \Delta$. 


\section{The $\left(G^{\prime} / G\right)$-expansion method}

The objective of this section is to use the $\left(G^{\prime} / G\right)$ expansion method [14] to solve the BBM-B Eq. (1). As we have done previously, we assume the solution to (1) can be expressed as a travelling wave

$$
u(x, t)=U(\xi), \quad \xi=x-\lambda t,
$$

where $\lambda$ is a constant and prime denotes the derivative with respect to $\xi$. Substituting $U(\xi)$ with wave variable $\xi$ in (1) then integrating once and setting the constant of integration to zero, we find

$$
\left(a-\lambda+\frac{b_{1}}{n+1} U^{n}+\frac{b_{2}}{2 n+1} U^{2 n}\right) U+c U^{\prime}-k \lambda U^{\prime \prime}=0 .
$$

In setting the constant of integration to zero does reduce the generality of the solution of (39). However, if $U(\xi)$ has at least one tail that asymptotically approaches zero, such as a shock wave, then setting the constant of integration to zero is perfectly reasonable. Periodic solutions are also possible although there is a loss of an arbitrary constant in the final solution [15]. Making the transformation $U(\xi)=f(\xi)^{\frac{1}{n}}$ in $(39)$ yields

$$
\begin{aligned}
& n^{2}\left(a-\lambda+\frac{b_{1}}{n+1} f+\frac{b_{2}}{2 n+1} f^{2}\right) f^{2}+c n f f^{\prime} \\
& \quad-k \lambda(1-n)\left(f^{\prime}\right)^{2}-n k \lambda f f^{\prime \prime}=0,
\end{aligned}
$$

where $f(\xi)$ is a real non-zero function.

According to the $\left(G^{\prime} / G\right)$-expansion method [14] the function $f(\xi)$ is expressed as a polynomial in $\left(G^{\prime} / G\right)$ :

$$
f(\xi)=\sum_{i=1}^{m} a_{i}\left(\frac{G^{\prime}}{G}\right)^{i}+a_{0}, \quad a_{m} \neq 0 .
$$

where $a_{i}(i=0,1, \ldots, m)$ are constants to be determined and $G(\xi)$ satisfies a second-order linear ordinary differential equation

$$
\frac{\mathrm{d}^{2} G(\xi)}{\mathrm{d} \xi^{2}}+\nu \frac{\mathrm{d} G(\xi)}{\mathrm{d} \xi}+\mu G(\xi)=0,
$$

where $\nu$ and $\mu$ are arbitrary constants. The general solutions to (42) are

$$
\frac{G^{\prime}(\xi)}{G(\xi)}=\left\{\begin{array}{l}
\frac{\sqrt{\beta}}{2}\left(\frac{C_{1} \sinh \left(\frac{\sqrt{\beta}}{2} \xi\right)+C_{2} \cosh \left(\frac{\sqrt{\beta}}{2} \xi\right)}{C_{1} \cosh \left(\frac{\sqrt{\beta}}{2} \xi\right)+C_{2} \sinh \left(\frac{\sqrt{\beta}}{2} \xi\right)}\right)-\frac{\nu}{2}, \quad \beta>0, \\
\frac{\sqrt{-\beta}}{2}\left(\frac{-C_{1} \sin \left(\frac{\sqrt{-\beta}}{2} \xi\right)+C_{2} \cos \left(\frac{\sqrt{-\beta}}{2} \xi\right)}{C_{1} \cos \left(\frac{\sqrt{4 \mu-\lambda^{2}}}{2} \xi\right)+C_{2} \sin \left(\frac{\sqrt{-\beta}}{2} \xi\right)}\right)-\frac{\nu}{2}, \quad \beta<0,
\end{array}\right.
$$

where $\beta=\nu^{2}-4 \mu$ and $C_{1}, C_{2}$ are arbitrary constants.

By considering the homogeneous balance between $f^{4}$ and $f f^{\prime \prime}$ in $(40)$ we get $4 m=2 m+2$, hence $m=1$. Then,

$$
f(\xi)=a_{0}+a_{1}\left(\frac{G^{\prime}}{G}\right), \quad a_{1} \neq 0 .
$$

Following Wang et al. [14], substituting (44) into (40) and using (42), we get the following constraints on the parameters:

$$
\mu=0,
$$

$$
\begin{aligned}
& \lambda=\frac{n(a n-\nu c)}{n^{2}+k \nu^{2}}, \\
& b_{1}=\frac{(n+1)\left(\nu k n a(n+2)-\nu^{2} k c(n+1)+c n^{2}\right)}{n\left(n^{2}+k \nu^{2}\right) \sqrt{\frac{k(n+1)(2 n+1)(a n-\nu c)}{n b_{2}\left(n^{2}+k \nu^{2}\right)}}}, \\
& a_{0}=0, \\
& a_{1}= \pm \sqrt{\frac{k(n+1)(2 n+1)(a n-\nu c)}{n b_{2}\left(n^{2}+k \nu^{2}\right)}}
\end{aligned}
$$

where $\nu \neq 0$ with $n, b_{2}, c, a, k$ being arbitrary. The solution is

$$
f(\xi)= \pm \sqrt{\frac{k(n+1)(2 n+1)(a n-\lambda c)}{n b_{2}\left(n^{2}+k \lambda^{2}\right)}}\left(\frac{G^{\prime}}{G}\right) .
$$

Since $\mu=0$, then hyperbolic functions only appear in the solutions

$$
\left(\frac{G^{\prime}}{G}\right)=\frac{\sqrt{\nu^{2}}}{2}\left(\frac{C_{1} \sinh \left(\frac{\sqrt{\nu^{2}}}{2} \xi\right)+C_{2} \cosh \left(\frac{\sqrt{\nu^{2}}}{2} \xi\right)}{C_{1} \cosh \left(\frac{\sqrt{\nu^{2}}}{2} \xi\right)+C_{2} \sinh \left(\frac{\sqrt{\nu^{2}}}{2} \xi\right)}\right)-\frac{\nu}{2} .
$$

From (45) we get the wrong impression that there are two arbitrary constants, $C_{1}$ and $C_{2}$. In fact there is only one, $C=C_{2} / C_{1}$ (or $\left.D=C_{1} / C_{2}\right)$. The solutions may be rewritten as

$$
\begin{aligned}
& f(\xi)= \pm \sqrt{\frac{k(n+1)(2 n+1)(a n-\nu c)}{n b_{2}\left(n^{2}+k \nu^{2}\right)}} \\
& \times\left[\frac{\sqrt{\nu^{2}}}{2}\left(\frac{\tanh \left(\frac{\sqrt{\nu^{2}}}{2} \xi\right)+C}{1+C \tanh \left(\frac{\sqrt{\nu^{2}}}{2} \xi\right)}\right)-\frac{\nu}{2}\right], \\
& f(\xi)= \pm \sqrt{\frac{k(n+1)(2 n+1)(a n-\nu c)}{n b_{2}\left(n^{2}+k \nu^{2}\right)}} \\
& \times\left[\frac{\sqrt{\nu^{2}}}{2}\left(\frac{1+C \operatorname{coth}\left(\frac{\sqrt{\nu^{2}}}{2} \xi\right)}{\operatorname{coth}\left(\frac{\sqrt{\nu^{2}}}{2} \xi\right)+C}\right)-\frac{\nu}{2}\right] .
\end{aligned}
$$

It is possible to further simplify these solutions

$$
\begin{aligned}
f(\xi) & = \pm \sqrt{\frac{k(n+1)(2 n+1)(a n-\nu c)}{n b_{2}\left(n^{2}+k \nu^{2}\right)}} \\
\times & {\left[\tanh \left(\frac{\sqrt{\nu^{2}}}{2}\left(\xi+\xi_{0}\right)\right)-\frac{\nu}{2}\right], } \\
f(\xi) & = \pm \sqrt{\frac{k(n+1)(2 n+1)(a n-\nu c)}{n b_{2}\left(n^{2}+k \nu^{2}\right)}} \\
\times & {\left[\operatorname{coth}\left(\frac{\sqrt{\nu^{2}}}{2}\left(\xi+\xi_{0}\right)\right)-\frac{\nu}{2}\right], }
\end{aligned}
$$

where

$$
\tanh \left(\frac{\sqrt{\nu^{2}}}{2} \xi_{0}\right)=C .
$$

These results show that there is both a topological soliton (shock wave) solution (48) and a singular soliton solution (49) available for the BBM-B equation. 


\section{Discussion}

We have used three seemingly different approaches in an attempt to find travelling wave solutions to the hybrid BBM-B equation (1). The ansatz method produced only one solution where as the Lie-symmetry method and the $\left(G^{\prime} / G\right)$-method produced multiple solutions. It is tempting to claim that these solutions are all new. Many have claimed this in previous studies of other nonlinear evolution equations (see for example [16-19]. Recently, Parkes [20, 21] and Kudryashov [22, 23] have criticised such claims. They showed convincingly that in most cases the new solutions are merely old solutions in disguise.

It is well known that for every tanh solution (27) (or (48)) there is a coth solution (28) (or (49)), being related via the substitution $\Delta \xi \rightarrow \Delta \xi-i \pi / 2$. Solutions of the tanh - coth type (31) are disguised coth solutions [21] and are related via the hyperbolic identity $2 \operatorname{coth}(\Delta \xi / 2)=\tanh (\Delta \xi / 4)+\operatorname{coth}(\Delta \xi / 4)$. Further, (29) can be expressed as (30) via $\Delta \xi \rightarrow \Delta \xi-i \pi / 2$, which in turn (30) can be reduced to (28) or (27) by using $\operatorname{coth}(\Delta \xi / 2)=\operatorname{coth}(\Delta \xi)+\operatorname{csch}(\Delta \xi)$ or $\tanh (\Delta \xi / 2)=$ $\operatorname{coth}(\Delta \xi)-\operatorname{csch}(\Delta \xi)$.

Also, Eqs. (34) to (37) are related and can be recast as either (27) or (28), this is most easily seen by setting $B=0$. Likewise, on setting $F=0,(32)$ and (33) can be reduced to (28) and (27), respectively.

However, the singular soliton solution (28) is not a bound solution and as such may have no physical meaning in the context of (1). The unbounded increase in the amplitude of the wave may be due to wave-breaking or filamentation, in either case signalling an instability of some kind. Moreover, solutions of the type (29) are clearly complex in contradiction to the requirement that $f(\xi)$ be real in both the Lie-symmetry method and $\left(G^{\prime} / G\right)$-method. As such these solutions must be discounted, however, often they are not (see [24-26] as illustrative examples).

\section{Conclusions}

We have derived and discussed the shock wave and other solutions for the dissipative shallow water wave equation governed by the BBM-B equation with dual power-law nonlinearity. The shock wave solution (27), often referred to as a topological soliton solution, is obtained using three approaches - the ansatz method, Liesymmetry method and the $\left(G^{\prime} / G\right)$-expansion method. The latter two approaches also provide singular soliton solutions (such as (28) or (49)) however these solutions are useful only if they are physical meaningful. We demonstrated that the multiple solutions obtained via the Lie-symmetry and $\left(G^{\prime} / G\right)$-method are not new solutions but disguised solutions of (27) and (28).

In relation to the shock wave solution of (1), solutions (2), (27), and (48) (for $n=1$ ) are essentially the same. This is not surprising as all three methods are reformulations of the well know tanh-expansion method
$[21,23]$. Any differences in the solutions are wholly attributable to the slightly different constraints that are applied in each case.

The ansatz method is the most direct and efficient of the three methods although using a symbolic computer package such as MAPLE or MATHEMATICA makes the calculations in all cases rather straightforward.

\section{Acknowledgments}

A. Biswas is grateful to the University of New South Wales (Canberra) for the funding of a visiting fellowship during April--June 2013, where some of this research was carried out.

\section{References}

[1] R. Abazari, Rom. Rep. Phys. 66, 286 (2014).

[2] M. Antonova, A. Biswas, Commun. Nonlin. Sci. Num. Simul. 14, 734 (2009).

[3] L. Girgis, A. Biswas, Appl. Math. Comput. 216, 2226 (2010).

[4] P. Razborova, H. Triki, A. Biswas, Ocean Eng. 63, 1 (2013).

[5] G.-W. Wang, T.Z. Xu, S. Johnson, A. Biswas, Astrophys. Space Sci. 349, 317 (2014).

[6] H. Zhao, Chaos Solitons Fractals 36, 1283 (2008).

[7] D.J. Huang, D.S. Li, H.Q. Zhang, Chaos Solitons Fractals 31, 586 (2007).

[8] A. Senthilkumar, Turk. J. Math. 37, 652 (2013).

[9] E. Yomba, Phys. Lett. A 372, 1048 (2008).

[10] L.-H. Zhang, Appl. Math. Comput. 208, 144 (2009).

[11] H. Triki, Z. Jovanoski, A. Biswas, Appl. Math. Inf. Sci. 8, 113 (2014).

[12] H. Triki, Z. Jovanoski, A. Biswas, Ind. J. Phys. 88, 71 (2014).

[13] R. Cherniha, V. Davydovych, J. Phys. A Math. Theor. 46, 185204 (2013).

[14] M. Wang, X. Li, J. Zhang, Phys. Lett. A 372, 417 (2008).

[15] D.R. Rowland, Z. Jovanoski, Phys. Rev. B 54, 13484 (1996).

[16] X.-J. Miao, Z.-Y. Zhang, Commun. Nonlin. Sci. Num. Simulat. 16, 4257 (2011).

[17] C. Bai, Phys. Lett. A 288, 191 (2001).

[18] A.-M. Wazwaz, Math. Comp. Simulat. 72, 1 (2006).

[19] I.E. Inan, Phys. Lett. A 371, 90 (2007).

[20] E.J. Parkes, Appl. Math. Comput. 217, 1749 (2010).

[21] E.J. Parkes, Appl. Math. Comput. 217, 1759 (2010).

[22] N.A. Kudryashov, Commun. Nonlin. Sci. Num. Simulat. 14, 3507 (2009).

[23] N.A. Kudryashov, Appl. Math. Comput. 217, 1755 (2010).

[24] Z.-Y. Yan, Int. J. Mod. Phys. C 14, 225 (2003).

[25] Z.-Y. Yan, Comp. Phys. Commun. 148, 30 (2003).

[26] S.A. Khuri, Chaos Solitons Fractals 20, 1037 (2004). 